\title{
Les honoraires d'un chirurgien de la Haute-Alsace en 1536
}

\author{
Par Ernest Wickersheimer, Schiltigheim
}

Le ms. 2241 (ancien All. 283) de la Bibliothèque Nationale et Universitaire de Strasbourg était primitivement relié avec un exemplaire du Feldtbuch der Wundartzney de Hans von GerssdorfF (Strasbourg, Hans Schott, 1528 , in $-4^{\circ}$ ) qui, après avoir appartenu à l'anatomiste strasbourgeois TноMAS LAUTH, entra, à la mort de celui-ci, en 1826, à la Bibliothèque de l'Académie de Strasbourg et est actuellement conservé à la Bibliothèque Nationale et Universitaire sous la cote R 101.298.

Ses vingt feuillets ont été, au XVI $\mathrm{X}^{\mathrm{e}}$ siècle, remplis par les premiers possesseurs du volume. On y voit surtout des recettes de médecine, mais aussi des notes personnelles, mentions de voyages et de maladies $\left(\right.$ fol. $1 \mathrm{r}^{\circ}$ ), comptes de dépenses pour achats de lingerie $\left(\right.$ fol. $\left.3 \mathrm{v}^{\circ}\right)$.

Les fol. $1 \mathrm{v}^{\circ}-3 \mathrm{r}^{\circ}$ et plus des deux tiers du fol. $3 \mathrm{v}^{\circ}$ sont de la même main. C'est une liste de créances où l'on distingue deux parties. Dans l'une (fol. 1 $\mathbf{v}^{\circ}-2 \mathbf{r}^{\circ}$ ), le nom du débiteur n'est accompagné que du montant de la dette et, à la marge, de l'abréviation «Dt» (dedit), chaque fois que le débiteur s'en est acquitté. Dans l'autre $\left(\right.$ fol. $\left.2 \mathrm{v}^{\circ}-3 \mathrm{v}^{\circ}\right)$, il y a généralement une mention de plus: celle de l'origine de la dette. Ce sont des honoraires pour soins donnés à des malades.

Voici le texte de cette seconde partie dont les alinéas ont été numérotés afin de faciliter les citations dans le commentaire qui suivra.

1. Item Hans Hessenn, schmitt knecht hatt gebenn ij g. von einner wünten am arm. Dt.

2. Item Cassper Egems, rebknecht hatt gebenn ij g. vonn einner wünden an einner hant. - Dt.

3. Item Michel Phefferlenns, rebknecht hatt gebenn viiij s. - Dt.

4. Item der bey Moritz Kieffer gediennt hatt, der kieffer knecht hatt gebenn x s. vonn einner hannt zu heillen. - Dt.

5. Item Hans Schültz hatt gebenn vij g.

6. Item die kellerinn von Kennsseim hatt geben xij s. von einner hant.

7. Item Jockkob, schmitt knecht hatt gebenn iiiij s. - Dt.

8. Item Jorg vonn Urbach hann ich geheylt inn eim verding an einner hant umb vij g. und dorann hatt er mir gebenn iiij g. - Dt.

9. Item ich han geheiltt Hans Schültzen zu Keyssersperg zum wünden und ein stich, sol gebenn viij g., doran han ich iiij g. - Dt. 
10. Item Cunrat Kieffers knecht hatt geben iiiij s. - Dt.

11. Item der schnider knecht Cunrats sol gebenn iiij s. - Dt.

12. Item Hans Karrless froü ein arm uss der stett, ij g. - Dt.

13. Item Kyrrim sin frow ein arm uss der stett, sol ij g. - Dt.

14. Item Füll Joerg ein arm uss der stet, sol j g.

15. Item Hans Jorg geheiltt ein wünd und das öüwg, soll geben ij g.

16. Item Jocop Bintel ein wünt uff dem arm, sol j g. - Dt.

17. Item Ulrich Kieffers froü ein arm entzwey sol gebenn ij g. - Dt.

18. Item Michel Hüssers knecht geheyltt, hatt im ein holtz ein fus zerschlagenn, sol gebenn ij g., j ort. - Dt.

19. Item Claüs Lügner ein kint geheilt am kopf, sol gebenn $\mathrm{j}$ g.

20. Item Deng Grampe sol gebenn von wegen Claüs Stocker j omen winn. - Dt.

21. Item Hansz Willenn, weber geheiltt ein wünt im kopff und ein mol die finger, ein mol ann einner hant das kintt. - Dt.

22. Item Jocop Spreng von eim hüntz bis zu heillen j omen winn. - Dt.

23. Item der kieffer, der Keysser ist mit mir iber kümmen und soll gebenn j g, - Dt.

24. Item Petter der bey Bastian Zornn gedient hat, ist mit mir iber kümmen unnd sol gebenn viiij s. - Dt.

25. Item Glad Hans, Diemmen stieffater sol gebenn j g. - Dt.

26. Item der Hertke von sinner wünden im kopff, sol gebenn iij g. - Dt.

27. Item Bastionn Zornnen knecht geheilt ein stich, soll gebenn vj s.

28. Item Hans uff der beckenn stübenn geheilt ein wünd im kopff und dar noch die finger mer uff der hant und sin frow ein hant uss der stet und das kint, dut ij g. - Dt.

29. Item der schaffner vonn Altspach geheillt am fus, dut ij g. - Dt.

30. Item Paüllüs Frickenn kellerrin geheilt an der brinnen, soll gebenn viiij s. - Dt.

31. Item Paüllüs Frickenn mutter geheillt am fus, ist mir vj s. - Dt.

32. Item Rot Pethonn, Simmon Morant schwoger an der brinnen, soll gebenn j g. - Dt.

33. Item Velltenn von Urbeis geheilt ann einner hant, soll gebenn $13 \mathrm{~g}$. und han im einnen monat essenn geben, sol er sünder bezallenn. - Dt.

34. Item Jocop Meyger geheilt ein wünd im kopff, xviij s. - Dt.

35. Item die kellerenn uff der rebleit stübenn von ein stullgang, vj s.

36. Item Cunrat Kemler an der brinnen geheilt und drenck gesotten, dut viiij s. - Dt.

37. Item von Cuntzenn von Altspach vonn der wünden im schenckel, xviij s.

38. Item Morant, der schwumacher knecht, ist v s.

39. Item Hans, der vor Berronn geschossenn ist worden, ist iber kümen umb ij g., ein ortt. Hat gebenn $\mathbf{j}$ g., iiij blapert.

40. Item Jocop Knüssel, ij g.

41. Item Frantz von Grensse, Pladenn dotchterman hat gebenn xij s. d. Ist noch vj s.

La plupart des cas traités relèvent de la pathologie externe: plaies ou affections non spécifiées de la main ou des doigts $(2,4,6,8,21,28,33)$, de la tête $(19,21,26,28,34)$, du pied $(18,29,31)$, du bras $(1,16)$, de la cuisse $(37)$, de l'œil (15), luxations du bras $(12,13,14)$ et de la main $(28)$, fracture du bras (17), morsure de chien (22), blessure par arme à feu (39). Il est question 
de constipation (35) et, trois fois $(30,32,36)$, d'une angine dite «brinnen $»^{1}$ dont nous savons d'autre part qu'en 1517 elle sévit cruellement à Mulhouse et à Bâle ${ }^{2}$. Le mot «stich » $(9,27)$, plutôt qu'à une piqûre s'applique probablement à une affection interne telle que la pleurésie ou la pneumonie que caractérise une douleur lancinante ${ }^{3}$.

Le praticien, sans doute un chirurgien, qui rédigea ces notes, est resté anonyme. Le lieu de sa résidence n'est pas nommé; il devait être de quelque importance puisqu'on y trouvait un poële de boulangers (28), un poële de vignerons (35) et aussi un bain, si toutefois c'est bien ainsi qu'il faille interpréter l'expression «im lerlenbat», employée pour désigner le domicile d'un débiteur au fol. $1 \mathbf{v}^{\circ}$ du manuscrit. Ses clients sont à Kaysersberg (9), à Alspach $(29,37)$, à Kientzheim (6), à Orbey (33), à Urbach, aujourd'hui Fréland (8); ses débiteurs à Ammerschwihr, à Kientzheim et à Orbey (fol. $\left.1 \mathrm{v}^{\circ}-2 \mathrm{r}^{\circ}\right)$.

Parmi ceux à qui il donne ses soins, il y a des gens de métier, un tisserand (21), un tonnelier (23), deux ouvriers forgerons $(1,7)$, deux ouvriers vignerons $(2,3)$, un ouvrier tonnelier (4), un ouvrier cordonnier (38), auxquels s'ajoutent quatre domestiques ou ouvriers sans indication de métier $(10,18,24$, $27)$, un «schaffner» ou régisseur (29) et trois personnes qualifiées de «kellerin» $(6,30,35)$, terme qu'on ne saurait mieux traduire que par «cellérière », bien qu'il ait souvent été pris dans un sens beaucoup plus large ${ }^{4}$.

La date du document est précisée par un événement historique contemporain, le siège de Péronne où un certain Hans avait été blessé d'un coup d'arme à feu (39). Le siège de Péronne par les Impériaux que commandait le comte de Nassau, prince d'Orange, commença le 12 août et fut levé tout juste un mois plus tard, le 11 septembre 1536.

Certains de ces relevés d'honoraires où le nombre de visites et de consultations n'apparaît point, concernent plusieurs individus appartenant à la même famille $(21,28)$. Il arrive que le chirurgien ait lui-même préparé un remède, faisant en quelque sorte fonction d'apothicaire: «drenck gesottenn» (36). Il arrive aussi que le patient ait pris pension chez lui; alors les frais de

${ }^{1}$ Grimm, Deutsches Wörterbuch, II, c. 325; M. Höfler, Deutsches Krankheitsnamen-Buch, München, Piloty u. Loehle, 1899 , in $8^{0}$, p. 165/6.

${ }^{2}$ Math. Mieg, Der Stadt Mülhausen Geschichte bis zum Jahr 1816, II (1817), p. 11; cf. E. Martin et H. Lienhart, Wörterbuch der elsässischen Mundarten, II (1904), p. 191.

${ }^{3}$ M. HöFLER, op. cit., p. 677 et s.

${ }^{4}$ Grimm, op. cit., V, c. 518/9. 
nourriture sont comptés à part $(33)^{5}$. Trois fois $(8,23,24)$, il est dit explicitement que les honoraires ont été fixés d'avance, par contrat.

Ces honoraires sont évalués soit en florins (gulden), soit en schillings ${ }^{6}$, désignés respectivement par les initiales «g» et «s». ${ }^{7}$

Les localités citées donnent lieu à penser qu'il s'agit du schilling colmarien dont la valeur, double de celle du schilling bâlois, était en 1536, d'environ 43 centimes or ${ }^{8}$. Quant au pouvoir d'achat, il aurait été approximativement de 23 fr., 54 or pour le florin de $12 \mathrm{sch} .1 / 2$ et de 1 fr. 87 or pour le schilling, si, ainsi que l'admet l'abbé HANAUER ${ }^{9}$, on l'obtient en multipliant la valeur numéraire par le cœfficient 4,36 .

A deux reprises $(20,22)$, il es dit que le chirurgien a accepté, en guise d'honoraires, une mesure de vin, «j omen winn».

A quoi cela répond-il ? Une ligne du fol. $1 \mathrm{v}^{\circ}$ nous l'apprendra. L'énoncé d'une dette: «Item Anstet Schermine iij g.» est suivie de cette note: «Hat geben ij omen win im jor 1537.» Deux mesures de vin, (la mesure étant d'un peu moins de 50 litres), pour 3 florins, cela met la mesure à 1 florin 1/2.

Il m'a paru intéressant de joindre à cette liste de créances un document qui en est assez proche, aussi bien dans l'espace que dans le temps.

A la date du 8 ou du 10 juillet (lundi ou mercredi avant la Sainte-Marguerite) 1566, on lit dans le «Ratsbuech zue Thann», au fol. $41 \mathrm{v}^{\circ}$ :

Rath montags mitwochen vor Margarethae anno 1566.

Meister JACOB REWER, der scherer fordert vonwegen heilung des Butschers von Altenthan bueben dem er REwER den schenckel abschneiden muessen, 12 gulden.

Sein ime 10 gulden geordtnet da soll auss Sannct Dieboltz ynkhomen ein dritteil unnd auss Sannct Erhartz gefellen der ander dritteil, sodan der Butsch (dweil der bueb auss seinem verworlosenn geschediget) den drittenteil bezalen.

\footnotetext{
${ }^{5}$ Autre exemple de chirurgiens ayant un malade en pension. Dans les comptes du chapitre de Saint-Maimbœuf de Montbéliard (1599-1600), figure une dépense de 30 livres accordées à Pierrelin Borne et Nicolas Lalouette, chirurgiens, pour avoir pansé et nourri un manœuvre, blessé par la chute d'une porte qu'il aidait à poser à la tour des Halles. Archives du Doubs, G. 1622.

6 Exceptionnellement est citée une autre monnaie: «blapert» (39), ou "plappert» dont la valeur dépassait légèrement 20 centimes or. - «Ort» $(18,39)$, veut dire un quart; «ij g., $j$ ort » se traduit par 2 florins $1 / 4$.

" Celle-ci remplacée (41) par la double initiale «s. d.» (solidus denarius = schilling pfennig).

8 Moyenne entre la valeur numéraire de 1533 (46 centimes) et celle de 1539 (40 centimes).

A. HANAUER, Etudes économiques sur l'Alsace ancienne et moderne, I (1876), p. 499.

9 A. Hanauer, op. cit., II (1878), p. 607, et du même, Guide monétaire pour l'histoire d'Alsace, dans Revue catholique d'Alsace, (1894), p. 784.
} 
Ainsi, en matière d'honoraires, les pouvoirs publics avaient leur mot à dire.

Pour une amputation de cuisse pratiquée sur le domestique ou l'apprenti de Butscher (ou Butsch) ${ }^{10}$, habitant de Vieux-Thann, le chirurgien JAcoB REWER réclamait 12 florins. Ceux-ci furent réduits à 10 , par décision du Magistrat de Thann. En outre il fut ordonné que pour un tiers cette somme serait prise sur les revenus du chapitre de Saint-Thiébaut, pour un autre tiers sur ceux de l'hôpital Saint-Erhart et que Butscher serait astreint à verser le troisième tiers, puisque c'était par suite de sa négligence que le garçon avait du être opéré.

10 Emule Herzog (Inventaire-sommaire des Archives municipales de la ville de Thann antérieures à 1790 ..., Colmar, impr. Alsatia, s. d., in $4^{0}$, p. 17) dit qu'il s'agit du fils de Butscher. La responsabilité pécuniaire imputée à Butscher ferait plutôt supposer qu'il était non le père, mais le patron de l'opéré. 